\title{
Evaluating the Advantages and Disadvantages of Teledermatology
}

\author{
Amit S. Kerure ${ }^{1}$, Nitika S. Deshmukh $\left(\mathbb{D}^{2,}{ }^{2}\right.$, Shaurya Rohatgi ${ }^{1}$ and Sandeep Agarwal ${ }^{3}$ \\ ${ }^{1}$ Consultant Dermatologist, Pune, India \\ ${ }^{2}$ Department of Skin VD, BJGMC, Pune, India \\ ${ }^{3}$ Department of Skin VD, IGGMC, Nagpur, India \\ "Corresponding author: Department of Skin VD, BJGMC, Pune, India. Email: nitikadesh15@gmail.com
}

Received 2020 November 15; Revised 2021 May 26; Accepted 2021 June 03.

Keywords: Cutaneous, Dermatology, Trichoscopy

\section{Dear editor,}

Telemedicine is defined as delivery of health care services, in remote areas or inaccessible areas or where distance is a crucial factor, by health care professionals using information and communication technologies for exchange of valid information for diagnosis and management of patients' diseases or for research purpose, all in the view of advancing the health of individuals (1).

Personal visit and physical consultation between the patient and the clinician remains the gold standard to provide appropriate clinical care to patients. There are some specific identifiable risks of online consultations, which are important, particularly during the outbreak of communicable diseases like coronavirus disease 2019 (COVID19) pandemic, where it is proposed that online consultation can be used as a supplementary method to consult patients rather than providing traditional physical consultation to the patient in non-emergency situations.

Any possible compromise in attribute of clinical assessment should be judged by the clinician and taken into consideration immediately, balancing the advantages and disadvantages of telemedicine with the firm ideal of positive and safe patient management in mind (Table 1).

Teledermatology is a double-edged sword. The most important concern about online consultation has been the accuracy of diagnosis, especially by conventionalists. Studies have shown that telemedicine has a diagnostic accuracy of 74 , and $61 \%$ of patients are diagnosed to have additional dermatoses during follow-up personal visits (2). On the contrary, according to another study by Lamel et al., where teledermatology was used as an interactive session, 70\% improvement was observed in patient management (3). Online dermatological consultation can be done in two ways, including store-and-forward (S\&F) and liveinteractive sessions (LI), the features of which have been il- lustrated in Table 2 (3).

Poor-quality photographs shared by the patient can significantly lower diagnostic accuracy and increase errors. An untrained person may not know which lesions to photograph, how to photograph, or may choose to ignore private parts, oral mucosa, etc. It is also difficult to take photos of areas not directly accessible to the patient, like back, buttocks, scalp, etc.

Hair pull test, global examination of scalp, trichoscopy, dermoscopy, histopathology, and routine bed side tests cannot be performed in online consultation setting, which may lead to diagnostic errors. Since online consultation depends so much on technology, there are opportunities for that technology to fail. Poor camera or inexperienced hands give poor image quality, which can impact a diagnosis. The patients who struggle with technology may find online consultation overwhelming and frustrating, which may impact their overall experience with the doctor. Moreover, the elderly may not take up or feel comfortable with the new technology, and the skin condition related to the genital area can be quite embarrassing.

Online consultation may be good for some diseases, but not for all of them. Some conditions that require a full body examination, like metastasis or surveillance for skin cancer, may be better suited for an in-person visit. There is risk of missing or delay in the diagnosis of incidental skin malignancy. Clinical photography for evaluation as well as documentation is not possible in teledermatology. Also, it is difficult to obtain specific consent required for further assessment by investigation or referral.

Inability to access patients' past data can be challenging, especially while managing unfamiliar patients. One cannot rely on a patient to accurately recollect their own history without access to notes, particularly in complicated cases. Through online consultation, inability to ex- 


\begin{tabular}{|c|c|}
\hline Consultation in Clinic & Consultation by Teledermatology \\
\hline $\begin{array}{l}\text { Dermatologist takes the history and asks further expert questions to explore the } \\
\text { clinical problem. }\end{array}$ & History taking may be done by a third party in any online consultation platform. \\
\hline $\begin{array}{l}\text { Patient can be examined thoroughly; skin lesions can be touched for diagnostic } \\
\text { accuracy. Even if it is not possible to touch in case of contagious pandemic } \\
\text { situations, a 3D view helps a better diagnosis. }\end{array}$ & Lesions need to be diagnosed by seeing 2D photos or videos. \\
\hline $\begin{array}{l}\text { Dermatologists consult the patient face-to-face, where additional information can } \\
\text { be obtained, thereby optimising patient care and likely concordance with } \\
\text { treatment. }\end{array}$ & $\begin{array}{l}\text { Virtual consultation may lack in gathering all the necessary information. Physical } \\
\text { examination cannot be performed, which is particularly essential for diagnosing } \\
\text { hair disorders and assessing the progress of the disease. Major illnesses with } \\
\text { systemic involvement are not suitable for teledermatology. }\end{array}$ \\
\hline $\begin{array}{l}\text { Other co-morbid conditions can be evaluated. Diagnosis and treatment of any } \\
\text { additional dermatoses can be done by examination of other body parts. }\end{array}$ & $\begin{array}{l}\text { Treatment is restricted to photos or videos provided by the patient. Detailed } \\
\text { examination is not possible. }\end{array}$ \\
\hline $\begin{array}{l}\text { Patient compliance is better when the doctor answers all the queries and explains } \\
\text { the treatment on a prescription paper. }\end{array}$ & $\begin{array}{l}\text { Compliance can be poor as online consultation lacks prescription explanation. } \\
\text { However, nowadays, some updated software and smart phone apps are available, } \\
\text { which can give details about medication in patient's language. }\end{array}$ \\
\hline \multirow[t]{2}{*}{ Documentation and clinical accountability are possible. } & $\begin{array}{l}\text { Documentations like consent, patient's photos, etc. are difficult to manage. } \\
\text { Patients' consent is essential before starting any online consultation as there is } \\
\text { sharing of pictorial information on digital platforms. }\end{array}$ \\
\hline & $\begin{array}{l}\text { A very good technology and infrastructure is required to visualise good image } \\
\text { quality and keep patient records }\end{array}$ \\
\hline
\end{tabular}

Table 2. Features of Store-and-Forward (S\&F) and Live- Interactive Sessions (LI) Types of
Store-and-Forward (S\&F)
Clinical information is recorded, stored, and then forwarded to the clinician. Real
time dialogue is not possible.

Diagnosis is solely based on stored information and available images. Further consultation and obtaining of details can be done via phone calls.

The physician can consult at any time.

Images should be of good quality, which requires high-quality cameras. Sending images online requires low bandwidth as compared to live video calling in an LI session.
Virtual consultation may lack in gathering all the necessary information. Physical examination cannot be performed, which is particularly essential for diagnosing systemic involvement are not suitable for teledermatology.

Treatment is restricted to photos or videos provided by the patient. Detailed examination is not possible. which can give details about medication in patient's language. Patients' consent is essential before starting any online consultation as there is A very good technology and infrastructure is required to visualise good image amine the patient means that a significant source of objective and valuable information is not stored. Hence, patient data recording and storage is important. Patients with comorbidities need to be dealt with caution in terms of treatment, and their investigations and reports need to be updated in the software system from time to time in order to proper management of complex illnesses.

Monitoring of patients who are on immunosuppressants and steroids is difficult via online consultation. For example, long-term steroid requires serial weight measurements, record of abdominal girth, and blood pressure monitoring, which is difficult in the online setting.

Patients may find it too convenient to directly contact the doctor via online platforms and may contact the doctor too frequently and often for trivial concerns; and revenue is not always guaranteed. In current trends, a patient requires a user-friendly mobile app to get connected to a dermatologist. Some third-party platforms retain a high percentage of the revenue, leaving a very low fee to the health care provider.

But there are some advantages of online consultation too. It is easy and convenient for both the patient and the physician to use it. Minor skin ailments which can be easily diagnosed on clinical photos can be easily treated via teledermatology. Safe medicines with minimum side-effects can be prescribed to the patients, which might provide symptomatic relief to the patient and help buy some time until the patient visits the clinic personally. Diseases like tinea versicolor, dermatophytoses, milder forms of acne, and other dermatoses, which do not have systemic involvement, can be comfortably managed on video consultation. Teledermatology has been proven to be extremely useful in current pandemic situation. For patients, who are on multiple and prolonged follow-up visits for their chronic skin ailments, teledermatology can help to continue their management. It can aid in exchanging laboratory reports, managing the drugs doses, and adding or subtracting any drugs as per the medical requirement of the patient. It also 
helps to keep a good digital record of the patient.

Before the COVID-19 pandemic, the official position of the authorities regarding telemedicine/online consultations was unknown. Some state medical councils did come out with strict opposition, and even Indian associations warned our members to refrain from indulging in online consultation. But suddenly the need of the times forced the authorities to accept what it demonized in the past. Moreover, guidelines have also been set up in this regard (1). Nevertheless, the authors wish to raise certain concerns with telemedicine in dermatology so that they are addressed by the association and/or authorities for continuous improvement. Live interactive sessions should be preferred over S\&F method in order to increase accuracy in diagnosis and enable patient counseling.

Also, rules and regulations for telemedicine should be formulated differently for each specialty. Dermatology, as a branch of visual diagnosis in most clinical scenarios, can be considered for telemedicine with specific restrictions on utility of immunosuppressants, steroids, and diseases associated with systemic complications. Minor skin ailments can be easily addressed by teledermatology, while major skin diseases do require personal clinical visits for proper evaluation. Specialties like pediatric medicine, general medicine, obstetrics and gynecology, surgery, and ophthalmology have their own set of limitations under telemedicine, which should be individualized in Medical council of India (MCI) rules and regulations.

Telemedicine is a fast-growing industry, and it is expected to grow in future. Dermatologists should be alert while dealing with a patient through a booming healthcare trend. In today's pandemic situation, teledermatology should be used as a supplementary mean rather than a substitute to face-to-face clinical practice to diagnose and treat patients.

\section{Footnotes}

Authors' Contribution: Concepts: Amit S. Kerure, Nitika S. Deshmukh, Shaurya Rohatgi, Sandeep Agarwal; Design: Amit S. Kerure, Nitika S. Deshmukh; Definition of intellectual content: Amit S. Kerure, Nitika S. Deshmukh, Shaurya Rohatgi, Sandeep Agarwal; Literature search: Amit S. Kerure, Nitika S. Deshmukh; Data acquisition: Amit S. Kerure, Nitika S. Deshmukh; Data analysis: Amit S. Kerure, Nitika S. Deshmukh, Shaurya Rohatgi, Sandeep Agarwal; Manuscript preparation: Amit S. Kerure, Nitika S. Deshmukh, Shaurya Rohatgi, Sandeep Agarwal; Manuscript editing: Amit S. Kerure, Nitika S. Deshmukh, Shaurya Rohatgi; Manuscript review: Amit S. Kerure, Nitika S. Deshmukh, Shaurya Rohatgi, Sandeep Agarwal; Guarantor: Amit S. Kerure, Nitika S. Deshmukh, Shaurya Rohatgi.
Conflict of Interests: There was no conflict of interests. Funding/Support: There was no funding/support.

\section{References}

1. Ministry of Health and Family Welfare and Niti Ayog. Telemedicine practice guidelines enabling registered medical practitioners to provide healthcare using telemedicine. India: Ministry of Health and Family Welfare and Niti Ayog; 2020. p. 1-43.

2. Foels R, Grewe S, James A. Assessing the diagnostic accuracy and patient outcomes of teledermatology consultations- one veterans' hospital experience. J Am Acad Dermatol. 2019;81(4):AB173. doi: 10.1016/j.jaad.2019.06.644.

3. Lamel S, Chambers CJ, Ratnarathorn M, Armstrong AW. Impact of live interactive teledermatology on diagnosis, disease management, and clinical outcomes. Arch Dermatol. 2012;148(1):61-5. doi: 10.1001/archdermatol.2011.1157. [PubMed: 22250233]. 